\title{
PLASMA SPRAYED RESISTORS
}

\author{
D. P. H. SMITH and J. C. ANDERSON \\ Materials Section, Electrical Engineering Department, Imperial College, London SW7, 2BT, U.K.
}

(Received February 13, 1980)

\begin{abstract}
A range of films of mixed composition, prepared by arc plasma spraying, has been investigated. Both free powder and powder in a plastic binder forming a rod have been used in the spray process. A variety of mixtures of insulators, metals and semiconductors has been examined and it is shown that resistivity/temperature curves for the specimens produced have in general two points of inflection, the temperatures of which vary with composition. This allows prediction of a composition giving low T.C.R. at room temperatures for any given metal/semiconductor combination. A qualitative theoretical model explaining this effect is presented. It has also been demonstrated that a conductor/insulator combination need not necessarily exhibit a sharp transition. This allows a broad range of resistance values to be obtained simply by varying composition.
\end{abstract}

\section{INTRODUCTION}

The preparation of thick-film resistors by arc plasma spraying (APS) of powders has been described in earlier papers. ${ }^{1,2}$ The advantage of this method is that the process is cheap and quick and the substrates used do not need to be the expensive alumina required by thick-film screen printing technology. In this paper we report on an investigation aimed at establishing the criteria for the design of APS resistors having a wide range of resistance with low T.C.R.'s.

\section{EXPERIMENTAL PROCEDURE}

\subsection{Spraying Procedure}

All resistors have been prepared by spraying at $400 \mathrm{~A}$ with a gas flow rate of $251 \mathrm{1} / \mathrm{min}$ using a powder feed hopper. The spraying distance was $5 \mathrm{~cm}$ and the substrates are rotated under the plasma on a $17 \mathrm{~cm}$ diameter drum at 500 r.p.m. The gun is translated during spraying at $420 \mathrm{~cm} / \mathrm{min}$. The substrates were soda-glass microscope slides and a bar pattern of resistors, each bar being $1.5 \mathrm{~cm}$ by $0.3 \mathrm{~cm}$, was produced by photo resist masking as described in previous papers, ${ }^{1,2}$

\subsection{Resistivity - Composition}

When spraying mixtures of powders of high and low resistivity it is important to have as smooth a resistivity versus composition curve as possible, with no sharp transitions in the resistivity regions of interest. This enables the correct composition for a particular resistivity material to be chosen with little error.

Theoretical considerations of this problem have in the past been based on statistically predicting the formation of discrete chains of highly conducting material in an insulating matrix, whereas in practice multiple contacts between chains are bound to exist. Scarisbrick ${ }^{3}$ considered the case of equal size conducting and insulating spheres whose size is very much smaller than the bulk. The probability of chain formation across a small (microscale) volume using a random walk is found to be

$$
P=V_{c}\left(V_{c}^{-2 / 3}\right)
$$

where $V_{c}$ is the volume fraction of the conducting material. These volumes which Scarisbrick took as spherical, are inter-connected to form a unit cube of the bulk material composed of multiply connected chains. The randomly arrayed chains are then resolved into three perpendicular directions (Cartesian coordinates) and thus the area of contact to any face of the unit cube is determined. This combined with the probability factor derived in Eq. (1) gives a resistivity corresponding to the volume fraction $V_{c}$

$$
\rho\left(V_{c}\right)=\frac{\rho_{c}}{V_{c} \cdot V_{c}^{-2 / 3} \cdot C^{2}}
$$

where $\rho_{c}$ is the resistivity of the highly conducting spheres and $C^{2}$ is the square cross-section of the 


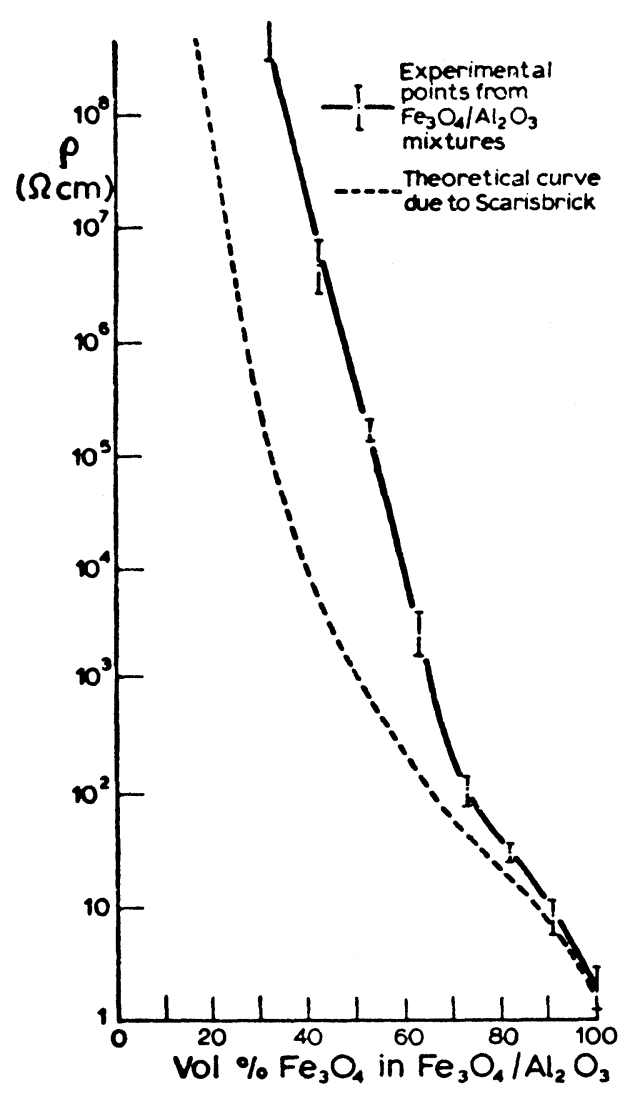

FIGURE 1 Resistivity v. volume fraction for $\mathrm{Fe}_{3} \mathrm{O}_{4} / \mathrm{Al}_{2} \mathrm{O}_{3}$ mixtures (1-3 $\mu \mathrm{m}$ particle size).

chains touching one face of the unit cube (which is also a function of $V_{c}$ ). This curve is plotted as the dotted line in Figure 1. In the plasma spraying process, the normal powder size is a distribution of roughly spherical, $10-90 \mu \mathrm{m}$ particles and these are delivered to the plasma gun by a gravity feed hopper. This method is unsuitable for spraying powders of less than $5 \mu \mathrm{m}$. Keeping the particle size appreciably smaller than the film thickness is necessary to meet the requirements of the theory given above and the thickness of the sprayed films used would not allow this to be done with particle size up to $90 \mu \mathrm{m}$.

A method of encasing fine powder in a polymer binder and forming this as a rod, which is subsequently fed into the plasma flame, has been tried and has met with moderate success. Only fairly refractory materials have been sprayed by this process and the films exhibit poorer adhesion than their hopper-fed counterparts. However a set of $\mathrm{Fe}_{3} \mathrm{O}_{4} / \mathrm{Al}_{2} \mathrm{O}_{3}$ powders (particle size $1-3 \mu \mathrm{m}$ ) has been sprayed by this method and the resultant resistivity versus composition curve is shown in Figure 1. As-sprayed $\mathrm{Fe}_{3} \mathrm{O}_{4}$ is a low resistivity semiconductor with a low activation energy of $0.09 \mathrm{eV}$. The deviation of the experimentally obtained curve from the theoretical may be due in part to the rather thin films $(10-15 \mu \mathrm{m})$ prepared for this study. The curve shows a higher resistivity than would be expected for a particular volume fraction of $\mathrm{Fe}_{3} \mathrm{O}_{4}$. Scarisbrick ${ }^{3}$ predicted this behaviour as the film thickness decreased towards a two-dimensional film. Despite this, the curve does represent a smooth variation of resistivity versus composition, although only high resistances are available $(>2 \mathrm{kohm})$ with very poor T.C.R. $(-12000$ p.p.m.) and poor adhesion.

When the particle sizes between the conducting and insulating phases differ, a form of threshold appears (see Figure 2). For the case of large conducting particles and small insulating particles an increased volume fraction of conducting material is necessary before continuous chains form and when they do the chain is of comparatively low resistivity (see

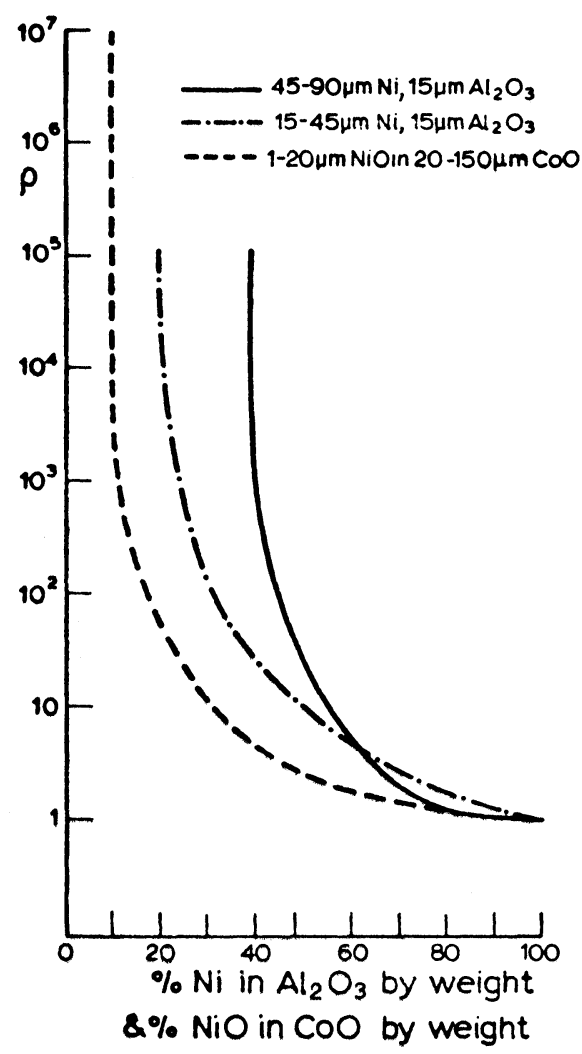

FIGURE 2 Resistivity $v$, volume fraction for metal/insulator mixtures of unequal particle sizes. 
the full curve in Figure 2). As the particle size ratio of metal to insulator is decreased to approximately three times, the threshold moves to a lower volume fraction with a corresponding increase in the resistivity at the threshold.

In the case of small metallic particles mixed with large insulating particles, the threshold occurs at a very small volume fraction and gives a high resistivity value. Subsequent increase in volume fraction of the metallic phase results in a rapid decrease in resistivity as the fine metallic particles surround the large insulating particles. Once this situation has been reached and the insulating particles. have been isolated, a conducting matrix is formed and the resistivity decreases fairly uniformly with volume fraction (see the dotted curve in Figure 2). These results are not surprising as similar behaviour has been observed elsewhere. ${ }^{4}$

In the case of a range of particle sizes for both the conducting and insulating species, the resulting curve is similar to the one shown for $\mathrm{NiO} / \mathrm{CoO}$ in Figure 2, (NiO partially reduces to form a dispersion of $\mathrm{Ni}$ ), i.e. for small metallic particles mixed with large insulating ones. A set of $\mathrm{Ni} / \mathrm{V}_{2} \mathrm{O}_{5} / \mathrm{Al}_{2} \mathrm{O}_{3}$ powders with particle sizes of 10-90 $\mu \mathrm{m}$ has been sprayed and has given such a curve.

This means that normal plasma sprayed powders are unsuitable for producing a smooth resistivitycomposition curve, but if the powders are carefully sieved (or prepared) to a fairly narrow particle size the sharp transition from high to low resistance over a narrow composition range can be avoided.

\subsection{T.C.R. of Composite Systems}

The T.C.R. of a simple system such as a metalinsulator mixture (e.g. $\mathrm{Ni}-\mathrm{Al}_{2} \mathrm{O}_{3}$ ) or a semiconductorinsulator system (e.g. $\mathrm{Fe}_{3} \mathrm{O}_{4}-\mathrm{Al}_{2} \mathrm{O}_{3}$ ) is determined solely by whether the threshold for continuous chains of the metal or semi-conductor has been reached. When this situation occurs the T.C.R. of the system is simply that of the metal $(\simeq 2400$ p.p.m. $)$ or the semiconductor $(\simeq 12000$ p.p.m. $)$ as would be expected.

In a more complicated system such as a metalsemiconductor mixture or a metal-semiconductorinsulator mixture the T.C.R.'s found experimentally exhibit a range of values from zero to the values given above for the individual metal or semiconductor (see Figures 3, 4 and 5). It can also be seen that in general two turning points (i.e. two points of zero T.C.R.) are found over the measured temperature range. The graphs are drawn as normalised

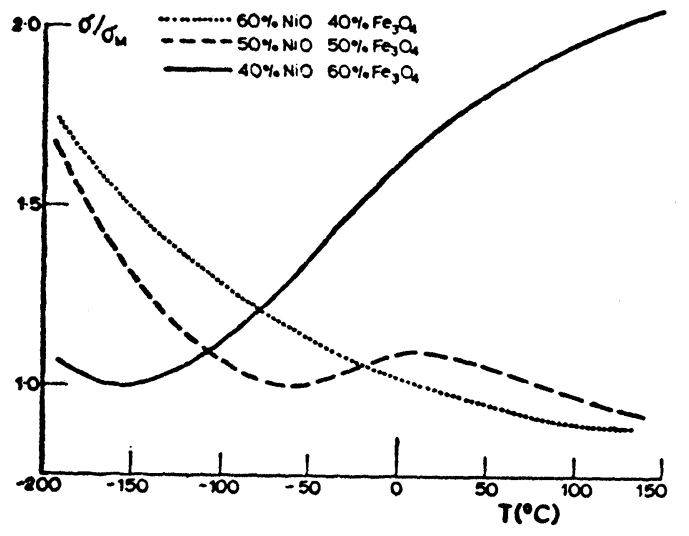

FIGURE 3 Normalised conductivity (with respect to conductivity minimum) v. temperature for $\mathrm{NiO} / \mathrm{Fe}_{3} \mathrm{O}_{4}$ mixtures.

conductivity curves in order to show all the curves in the same diagram. In order to understand this behaviour fully, a complete description of the possible particle shapes that occur in plasma sprayed films as well as the particle size distribution and probabilities of chain and near chain formation are needed. Work is continuing along these lines, but for a qualitative prediction of the kind of behaviour that might be expected, let it be assumed for simplicity that the particles are cubes of $10 \mu \mathrm{m}$ sides. The resistance of a purely metallic chain is

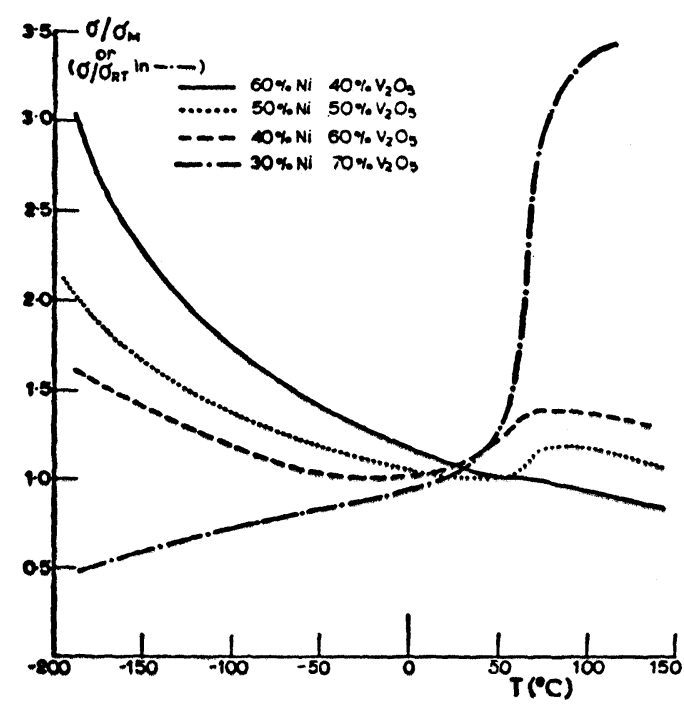

FIGURE 4 Normalised conductivity (with respect to conductivity minimum) v. temperature for $\mathrm{Ni} / \mathrm{V}_{2} \mathrm{O}_{5}$ mixtures. 


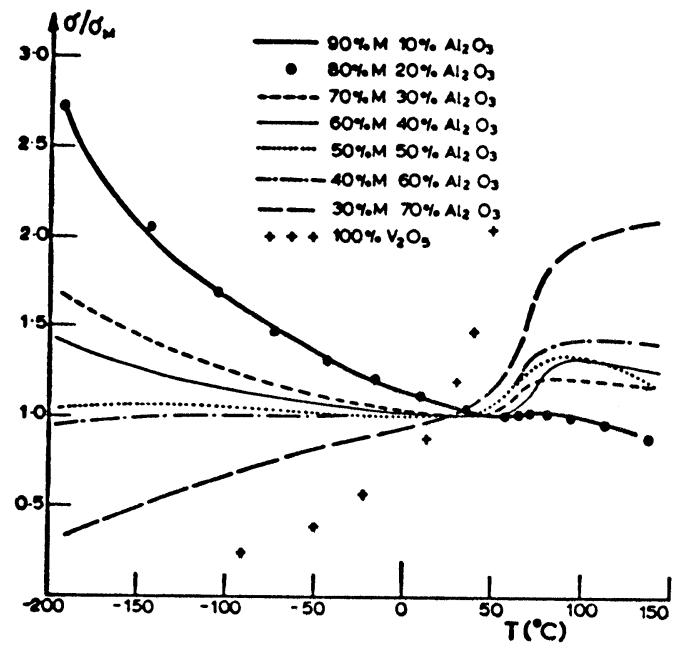

FIGURE 5 Normalised conductivity (with respect to conductivity minimum $) \mathrm{v}$. temperature for $\mathrm{M}(=60 \%$ $\mathrm{Ni} / 40 \% \mathrm{~V}_{2} \mathrm{O}_{5}$ by weight) $/ \mathrm{Al}_{2} \mathrm{O}_{3}$ mixtures.

given by

$$
R=n R_{m_{0}}(1+\alpha T)
$$

where $\alpha$ is the T.C.R. and $n$ is the number of cubes in one chain (this has been taken as 2500 , but the value does not affect the argument).

Now the conductivity is given by

$$
\sigma=\frac{l}{R a}
$$

where $l$ is the length and $a$ is the cross-sectional area of the chain.

For $r$ cubes of semiconductor in a metal chain the resistance is given by

$$
R_{r}=(n-r) R_{m_{0}}(1+\alpha T)+r R_{s_{0}} e^{\frac{E}{k T}}
$$

where $E$ is the activation energy of the semiconductor.

The conductivity curves for metal chains with zero, one, three and ten semiconducting particles in them are shown in Figure 6 for $E=0.09 \mathrm{eV}$, the value for $\mathrm{Fe}_{3} \mathrm{O}_{4}$.

In order to obtain the total conductivity of the system these expressions may be added together and the total curve is shown in Figure 6. This exhibits the two turning points that occur in most of the films experimentally. In fact this hypothesis leads to the conclusion that another minimum occurs at very high temperatures, when the pure semiconductor matrix becomes very conducting, but

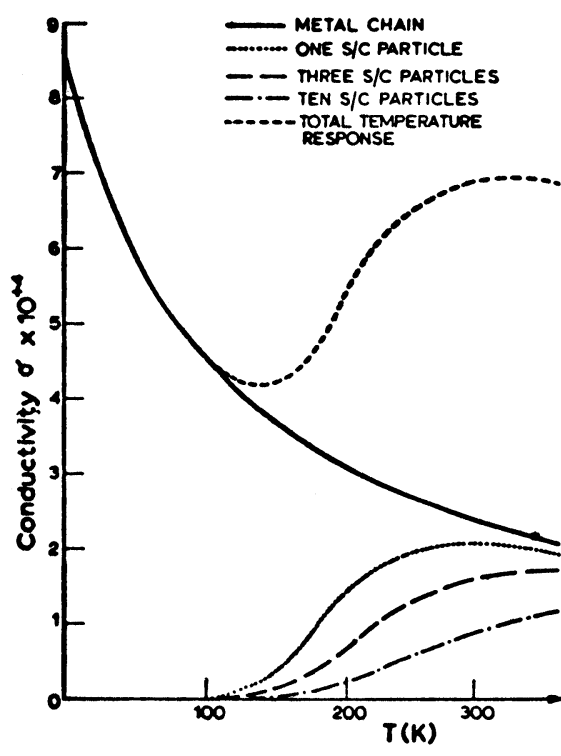

FIGURE 6 Qualitative model of conductivity v. temperature for a metal/semiconductor mixture.

this occurs at much too high a temperature to be observed.

Figures 3 and 4 support this hypothesis qualitatively and in fact more examples of this behaviour have been observed but are not reported here. In Figure 5 the mixture $M$ is in fact a $60 \%$ $\mathrm{Ni} / 40 \% \mathrm{~V}_{2} \mathrm{O}_{5}$ mixture by weight, which is subsequently mixed with $\mathrm{Al}_{2} \mathrm{O}_{3}$. The reason for the shift in minima towards lower temperature as the proportion of $M$ is decreased may be assigned to the non-linearity in the resistivity-composition curves, that is that at low compositions of $\mathrm{Ni}$ there is a steep dependence of resistivity on composition. The T.C.R.'s in Figure 5 vary from 100 p.p.m. to 2000 p.p.m. at room temperature, but the resistance range is only $0.5 \mathrm{ohm}$ to $1 \mathrm{kohm}$. However this series has a particle size range of 10-90 $\mu \mathrm{m}$, which has been concluded to be a poor choice as far as resistivity-composition is concerned.

\section{DISCUSSION AND CONCLUSIONS}

From the foregoing it can be deduced that APS films of good T.C.R. with a wide resistance range could be produced if certain design rules are observed. If equal, small-sized particles (say in the range $10-15 \mu \mathrm{m}$ ) of a high resistivity, low T.C.R. metal and an insulator were used a range of resistors having the T.C.R. of the metal could be produced. 
For example, using nichrome which has a T.C.R. of 180 p.p.m., resistivities up to $50 \mathrm{kohm} / \square$ could be obtained with film thicknesses down to $10 \mu \mathrm{m}$. For higher resistivities or for lower resistivities requiring a better T.C.R., a ternary metalsemiconductor-insulator system would be required. In this case the desired resistivity with a turning point at room temperature (for zero T.C.R.) may be determined experimentally by an iterative method. The addition of the semiconductor to the metal/insulator mixture will give a lower T.C.R. but also reduces the resistivity. A subsequent increase in the volume fraction of insulator in order to compensate for the lowered resistivity will then shift the zero point of the T.C.R., so that a further alteration of the metal/semiconductor volume fraction ratio will have to be made, and so on.
Using a more detailed theoretical model the number of experimental iterations could be considerably reduced. This model is at present being developed and will be the subject of a separate paper.

\section{ACKNOWLEDGEMENTS}

One of the authors (D. P. H. Smith) acknowledges the support of the Science Research Council via a Studentship Award during the work.

\section{REFERENCES}

1. R. T. Smyth, and J. C. Anderson, Electrocomp. Sci. and Technol., 2, (1975) 135-145.

2. R. T. Smyth, D.I.C. thesis, London University (1975).

3. R. M. Scarisbrick, J. Phys. D, 6 (1973) 2098.

4. A. Malliaris, and D. T. Turner, J. Appl. Phys. 42, (2) (1971) 614 

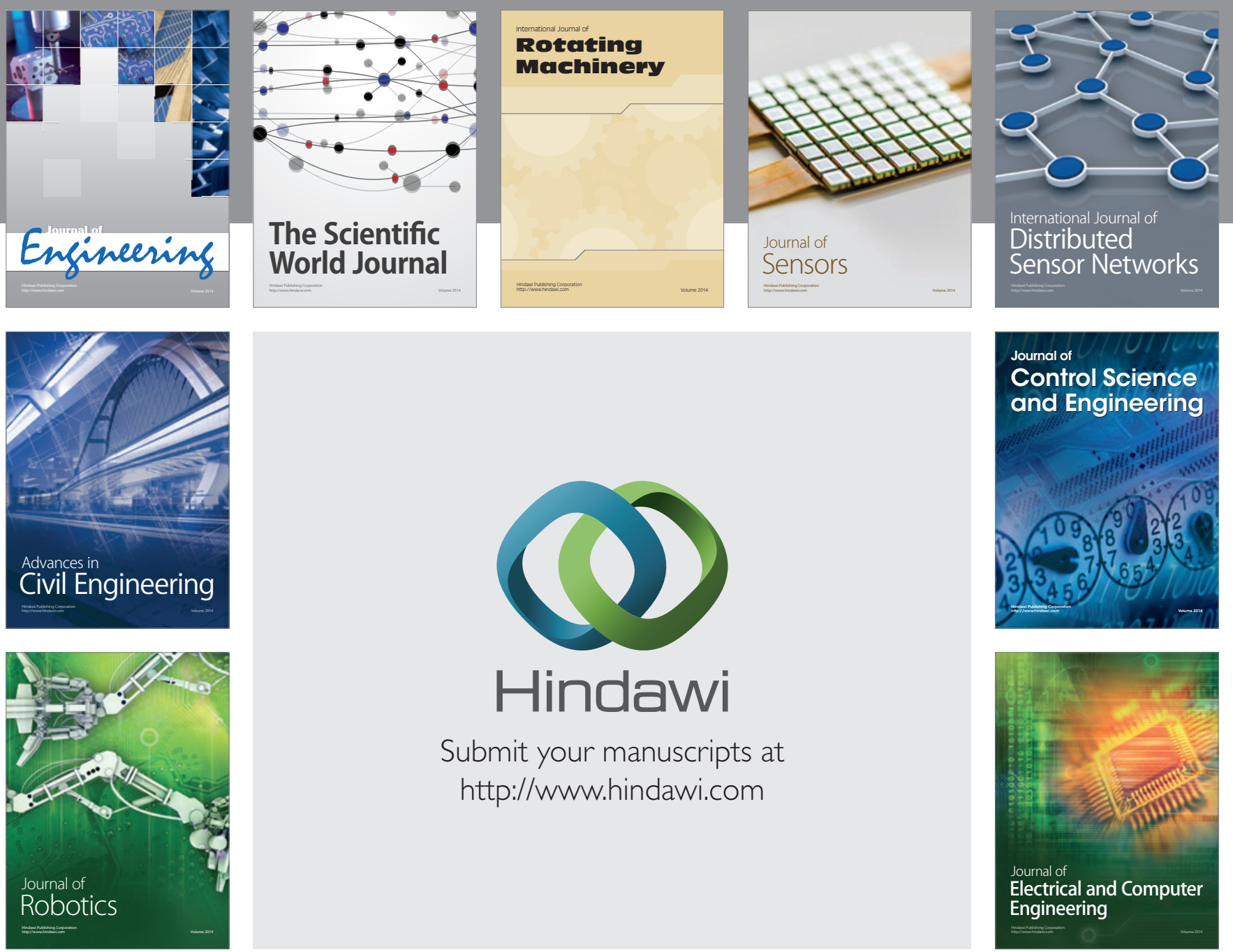

Submit your manuscripts at

http://www.hindawi.com
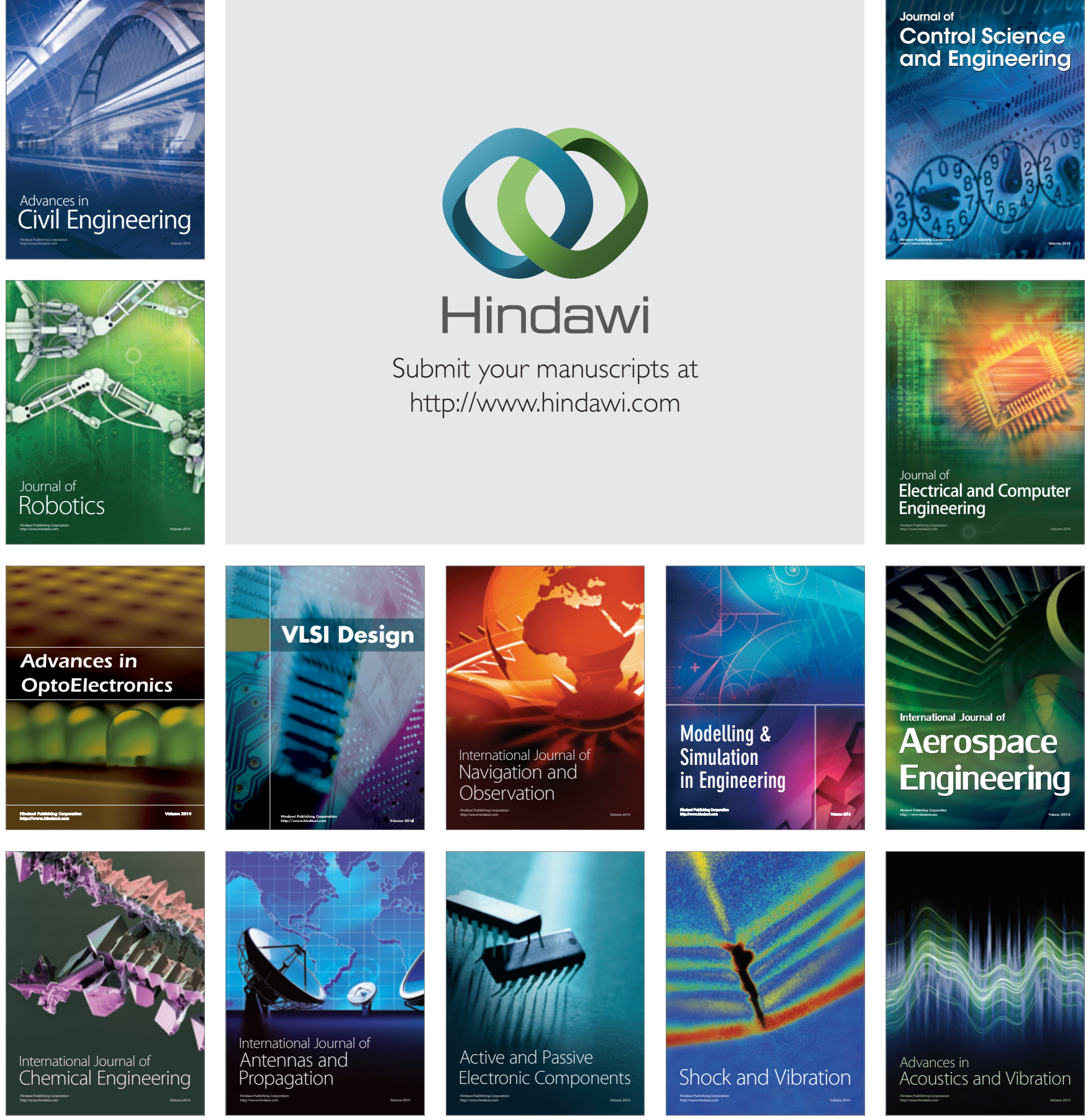